\title{
Pattern of Paresis in ALS is Consistent with the Physiology of the Corticomotoneuronal Projections to Different Muscle Groups
}

Albert C. Ludolph ${ }^{1}$, Susanne Emilian ${ }^{1}$, Jens Dreyhaupt ${ }^{2}$, Angela Rosenbohm¹, Alexander Kraskov ${ }^{3}$, Roger N. Lemon ${ }^{3}$, Kelly Del Tredici ${ }^{4}$, Heiko Braak ${ }^{4}$

${ }^{1}$ Department of Neurology, University of Ulm, Ulm, Germany

2Institute of Epidemiology and Medical Biometry, University of Ulm, Ulm, Germany

${ }^{3}$ Department of Clinical and Movement Neurosciences, UCL Queen Square Institute of Neurology, London, United Kingdom

${ }^{4}$ Clinical Neuroanatomy Section, Department of Neurology, Center for Biomedical Research, University of Ulm, Germany

Running title: Paresis in ALS

\section{Correspondence:}

Prof. Albert C. Ludolph, MD

Department of Neurology

University of Ulm

89075 Ulm

Phone: 0049 731-1771200

Fax: 0049 731-1771202

Email: albert.ludolph@rku.de

\section{Abstract}


Objective: A recent neuroanatomical staging scheme of amyotrophic lateral sclerosis (ALS) indicates that a cortical lesion may spread, as a network disorder, both at the cortical level and via corticofugal tracts, including corticospinal projections providing direct monosynaptic input to $\alpha$-motoneurons. These projections are involved preferentially and early in ALS. If these findings are clinically relevant, the pattern of paresis in ALS should primarily involve those muscle groups that receive the strongest direct corticomotoneuronal (CM) innervation.

Methods: In a large cohort ( $\mathrm{N}=436)$, we analyzed retrospectively the pattern of muscle paresis in ALS patients using the UK Medical Research Council (MRC) scoring system; we subsequently carried out two independent prospective studies in two smaller groups ( $\mathrm{N}=92$ and $\mathrm{N}=54)$.

Results: The results indicated that a characteristic pattern of paresis exists. When pairs of muscle groups were compared within patients, the group known to receive the more pronounced CM connections was significantly weaker. Within patients, there was greater relative weakness (lower MRC score) in thumb abductors vs. elbow extensors, for hand extensors vs. hand flexors and for elbow flexors vs. elbow extensors. In the lower limb, knee flexors were relatively weaker than extensors, and plantar extensors were weaker than plantar flexors.

Conclusions: These findings were mostly significant $(p<0.01)$ for all six pairs of muscles tested, and provide indirect support for the concept that ALS may specifically affect muscle groups with strong $\mathrm{CM}$ connections. This specific pattern could help to refine clinical and electrophysiological ALS diagnostic criteria and complement prospective clinico-pathologic correlation studies.

Keywords: amyotrophic lateral sclerosis, corticospinal tract, monosynaptic transmission, neuropathological staging, primates 


\section{Introduction}

As previously shown in Alzheimer's and Parkinson's diseases, a recent staging approach to sporadic amyotrophic lateral sclerosis (ALS) using the molecular marker phosphorylated 43-kDa TAR DNA-binding protein (pTDP-43) suggests that the pathology progresses, as a network disorder, across specific regions of the brain and spinal cord according to a predictable sequential anatomical pattern. ${ }^{1-8}$ Corticofugal tracts appear to be affected in a stereotypical pattern and, notably, involve targets receiving monosynaptic projections from the cortex, suggesting that these direct connections may preferentially propagate the disease process. ${ }^{5-9}$ This is particularly true for bulbar and spinal $\alpha$-motoneurons, the excitation of which is directly influenced by descending corticospinal and corticobulbar projections. ${ }^{10} 11$

Clinically, this pattern of pathology in sporadic ALS implies that muscle groups supplied by $\alpha$-motoneurons with strong CM connections should be more prone to functional loss and amyotrophy in ALS patients than are muscle groups whose $\alpha$-motoneurons receive less direct corticofugal connections.

The existence and distribution of corticomotoneuronal (CM) connections is of considerable importance in the phylogenetic development of hand skill across primate species. ${ }^{10,11}$ As such, direct control by the motor cortex of intrinsic and extrinsic hand muscles is unique to dexterous primates. ${ }^{11-13}$ The existence of direct CM connections has been established by both anatomical and electrophysiological investigations in non-human primates, and these studies have shown that a number of different muscle groups receive these projections, which, for the upper limb, are strongest to intrinsic muscles of the hand, but also involve wrist extensors and more proximal arm muscles, such as biceps brachii. ${ }^{10,11,14,15}$. The existence of direct CM connections to these muscle groups and to muscle groups in the lower limb has been confirmed in humans using non-invasive stimulation of the motor cortex. ${ }^{16-19}$, 19a, 19b The strength of CM influence over a muscle depends upon the number of $\alpha$-motoneurons contacted and the average size of the individual synaptic inputs. ${ }^{10}$

In clinical terms, after lesions of the central motor pathways (e.g., "Wernicke-Mann paralysis"20), the majority of motor deficits occur in the extremities, and these deficits 
display characteristic patterns of paresis $26 \mathrm{~b}, 26 \mathrm{c}$ characterized by weakness of the intrinsic hand muscles, the extensors of the hand and fingers, the biceps brachii muscles, and, in Wernicke-Mann syndrome, a preferential paralysis of the knee flexors and plantar extensors. It is unlikely, however, that clinical observations of "central patterns of paresis" reflect a lesion of the corticospinal pathway alone. Rather, such patterns may also represent the contribution of other descending tracts of subcortical origin, all of which also receive corticofugal fibers. ${ }^{10,11,15,21,22}$

Here, we retrospectively analyzed a cohort composed of 436 ALS patients, in whom we addressed the hypothesis that a pattern of muscle weakness exists that reflects the influence of direct CM connections. In addition, we attempted to test this hypothesis in two independent, smaller, prospective cohorts consisting of 92 and 54 ALS patients.

We hypothesized that

- if the lesions have disrupted CM connections to the upper extremities, the most affected (weakest) muscle groups should be the intrinsic hand muscles, with hand extensors being more affected than the hand flexors, and the biceps brachii muscles more than the triceps brachii muscles, whereas,

- in the lower extremities, the knee flexors (biceps femoris muscles) should be more affected (weaker) than knee extensors, and the plantar extensors (e.g., tibialis anterior) more severely affected than the plantar flexors (e.g., gastrocnemius and soleus muscles) (Fig. 1). 


\section{Patients and Methods}

\section{Patients}

The retrospective and prospective parts of the study were approved by the Ethics Review Board of the University of UIm (No. 11/10). Informed written consent had been provided by all patients.

\section{Pattern of muscle weakness (paresis)}

In a total of 436 patients from the Department of Neurology at the University of UIm, loss of muscle strength, as measured by the Medical Research Council (MRC) scale, ${ }^{23}$ was compared following physical examination and testing of strength in muscles or muscle groups known to be strongly influenced by CM projections, with muscles or muscle groups with less direct connections from the motor cortex (Fig. 1). The comparisons labelled V1, V2, V5 and V6 compared muscle groups acting at the same joint (elbow, hand, knee and ankle, respectively). The comparisons labelled V3 and V4 compared upper limb muscle groups known to receive some of the strongest CM projections (thumb abductors, hand extensors) with those that receive little or no CM input (elbow extensors). 


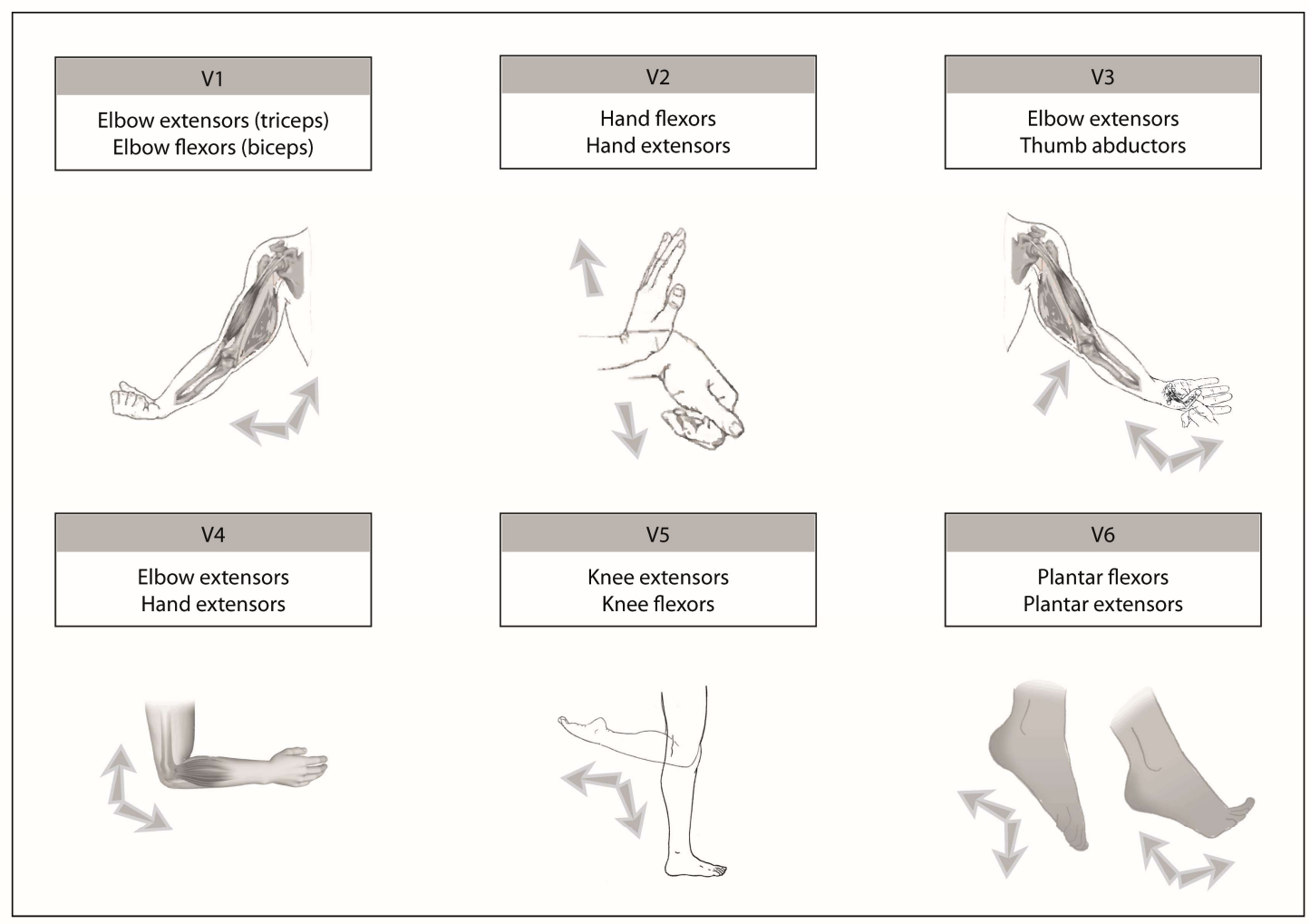

Figure 1. Muscle groups tested (V1-V6). The strength of muscle groups known to be more strongly influenced by CM projections was compared with those with less direct connections from the motor cortex. Strength of muscles was compared using the MRC score.

We first retrospectively analysed all available records of 436 patients from our data bank (2015/2016), which is based on a hospital-based register with a capturerecapture rate of $>80 \% .{ }^{24} 61 \%$ were male and $39 \%$ female, mean age of onset was 56.5 years, and median survival was 45.5 months. Forty-two (9.6\%) had a positive family history, $24 \%$ had bulbar and $76 \%$ spinal onset. Each patient met the El Escorial criteria for ALS, including possible ALS. ${ }^{25}$ If several test sessions took place, the first examination was used and all muscle groups were tested on this occasion.

In a subset of these patients $(\mathrm{N}=233)$, ALS clearly affected at least one muscle group in each pair, i.e., all four limbs showed evidence of ALS. This subset was analysed separately. The remaining patients 203 patients exhibited full strength (MRC 5) in each tested pair in at least one of four limbs. 
The second, independent, prospective study was carried out by a trained physiotherapist in 92 in- or outpatients of the Department of Neurology, University of Ulm. Fifty-two (56\%) were male, 40 (44\%) were female. Average age of onset of disease was 59.1 years; of this group 80 were still alive at the time of analysis, while 12 died after an average survival time of 29.2 months. Eight (9\%) had familial disease. Thirty-one patients (33\%) suffered from bulbar onset disease, the remaining had spinal onset. El Escorial criteria (including possible ALS) were met by each patient. Again, a separate analysis was carried out in a subset of these patients $(\mathrm{N}=61)$ in which ALS clearly affected at least one muscle group in each tested pair.

The third - prospective - study was done by an experienced neurologist (ACL) and included 54 patients fulfilling the El Escorial criteria, including possible ALS, 41 were male and 13 female. Mean age of patients at onset was 58.1 years, four had a positive family history, 17 had bulbar onset. A separate analysis in a subset of these patients in which ALS clearly affected at least one muscle group in each tested pair was not performed because of the small number of patients $(\mathrm{N}=11)$.

\section{Statistics}

For statistical analysis, the MRC measurements of muscle strength and differences of MRC measurements across pairs of muscle groups (as shown in Fig. 1) were described by absolute frequencies and percentages. Bar charts were used to compare the MRC measurements of strength in the nine muscle groups tested (five upper limb and four lower limb). A two-step procedure was applied for statistical analysis of MRC measurements. First, differences between the same muscle group on the right and left side in each limb were investigated using the two-sided sign test. The result of this first step showed no obvious differences between the right and left side. Thus, the MRC measurements from the right and left side were pooled and analysed collectively to obtain more power (second step).

In addition, the two-sided sign test was used to investigate differences between the pairs of muscle groups shown in Fig. 1. A result was considered as significant if the pvalue was $<0.05$. To account for multiple testing, the Bonferroni adjustment was applied to each p-value obtained from the second step. Statistical analyses were 
performed using SAS, version 9.4 (SAS Institute, Chicago, IL, USA). Figures were created using Matlab R2018b.

\section{Results}

\section{Pattern of paresis and differences in MRC grade across pairs of muscle groups: Retrospective study (436 patients)}

The 3D plot in Fig. 2A show the percentage distribution of individual MRC measurements for the nine muscle groups tested in the different cohorts. The results show that, in around $45-55 \%$ of patients, some muscle groups (elbow extensors, knee extensors and plantar flexors) retained full strength (MRC grade 5). This pattern was rarer for hand thumb abductors and hand extensors, and more patients exhibited the lowest MRC scores (0.1) in these same groups of muscles and in plantar extensors than in others tested.

In each of the 872 limbs in this retrospective cohort, we made a statistical comparison of the MRC grade for the 6 pairs of muscle groups shown in Fig. 1, using a two-sided sign test. Figure 2B plots the frequency of differences found. For example, for the V2 comparison, the same strength was found for both hand flexors and extensors in 702 limbs (80.5\%), the flexors were stronger in 137 limbs (15.7\%), but the extensors were stronger in only 33 limbs (3.7\%).

Although the median difference was zero for each pair tested (the large grey central bin in each of the plots), the data show that there was a significant difference $(p<0.01)$ in the distribution, with a larger proportion of limbs showing positive (red bars) than negative (blue) differences, indicating that the muscle group receiving the less pronounced CM projection was the stronger in each group pair tested. The skewed distribution was particularly pronounced for the elbow extensor-thumb abductor (V3) and for the plantar flexor-extensor (V6) comparison. 



Figure 2. Retrospective study in $\mathrm{N}=\mathbf{4 3 6}$ patients ( $872 \mathrm{limbs}$ ). A. Percentages of individual MRC scores for each muscle group. Bars indicate the percentage of MRC scores between 0 and 5 . Colour scale is autoscaled to the maximum MRC score across all muscle groups. B. Difference in MRC score between pairs of muscles for each muscle group. Bars indicate the percentage distribution of differences in MRC score. A positive difference (red bars) indicates that the muscle group receiving the less pronounced $\mathrm{CM}$ influence (first muscle group listed for each pair) was the stronger. Blue bars indicate in a difference in the opposite direction. The $p$ value derived from the sign test of these differences is given at the top of each panel.

\section{Retrospective study - only patients with all extremities affected (subset of 233 patients)}

We further analyzed the data from a subset of 233 patients in whom, in each limb, at least one muscle from the tested groups had a score of $<5$. Figure $3 \mathrm{~A}$ shows that results similar to those in the whole cohort were obtained, although - not surprisingly - differences were more pronounced. Once again the most marked weakness was in the plantar extensors, thumb abductors and hand extensors. Compared with Fig. 2A, a different viewing angle has been chosen for the data in Fig. $3 \mathrm{~A}$ to reveal the lower percentage of muscle groups with normal (MRC 5) scores in this subset of patients.

Figure 3B shows that again, although the median difference in MRC score was again zero for each pair of muscle groups, there was a clear and significant bias for every 
group towards positive differences, indicating the greater strength of the muscle groups that receive less pronounced CM inputs. The largest difference was seen between elbow extensors- thumb abductors (V3): Only $43.8 \%$ had the same score, while in $46.6 \%$ the thumb abductors were weaker. The difference between hand flexors and extensors was also comparatively large (V2, 73.0\% with the same score, but extensors weaker in $20.9 \%$ ), as was the difference in muscle strength between plantar flexors and extensors (V6, 59.9\% with the same score, extensors weaker in $35.8 \%$ ). The difference between knee extensors and flexors (V5) was smaller but still significant.
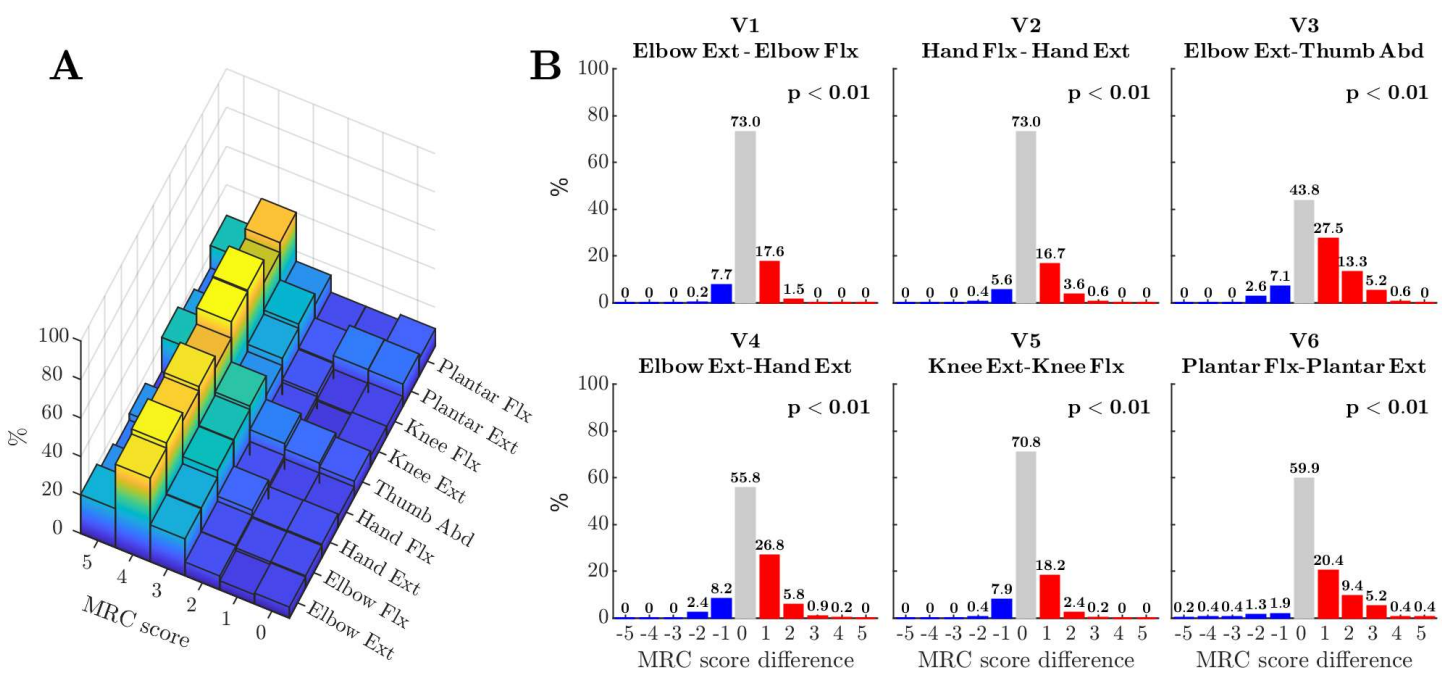

Figure 3. Retrospective study, only patients in whom all 4 limbs were affected (subset of $\mathbf{N}=233$ patients; $\mathbf{N}=184$ limbs). A. Percentages of individual MRC scores for each muscle group. B. Difference in MRC score between pairs of muscles for each muscle group. Bars indicate the percentage distribution of differences in MRC score. See legend accompanying Fig. 2 for further details.

\section{Pattern of paresis and differences in MRC grade across pairs of muscle groups: Prospective study (92 patients)}

To further test and substantiate our findings, a total of 92 hospitalized ALS patients was examined by an experienced physiotherapist (SE) using the MRC scale. Figure 4A shows the pattern of weakness across the nine muscle groups. The same pronounced differences were found, with substantially fewer patients with MRC grades 5 and 4 in the plantar extensors, thumb abductors, hand extensors and elbow flexors. 
The differences in muscle strength (Fig. 4B) were also similar, and these differences were mostly positive and were significant for the $\mathrm{V} 2, \mathrm{~V} 3, \mathrm{~V} 4$, and $\mathrm{V} 6$ pairs of muscle groups. Thus the results resembled those from the retrospective study performed in 436 individuals, with the exception of the proximal muscle groups: The difference between elbow extensors and flexors (V1) and between knee extensors and flexors (V5) failed to achieve the significance found in the retrospective studies. The difference may be accounted for by the fact that this second patient group consisted of individuals in an earlier stage of the disease who were in the hospital for early diagnostic procedures.
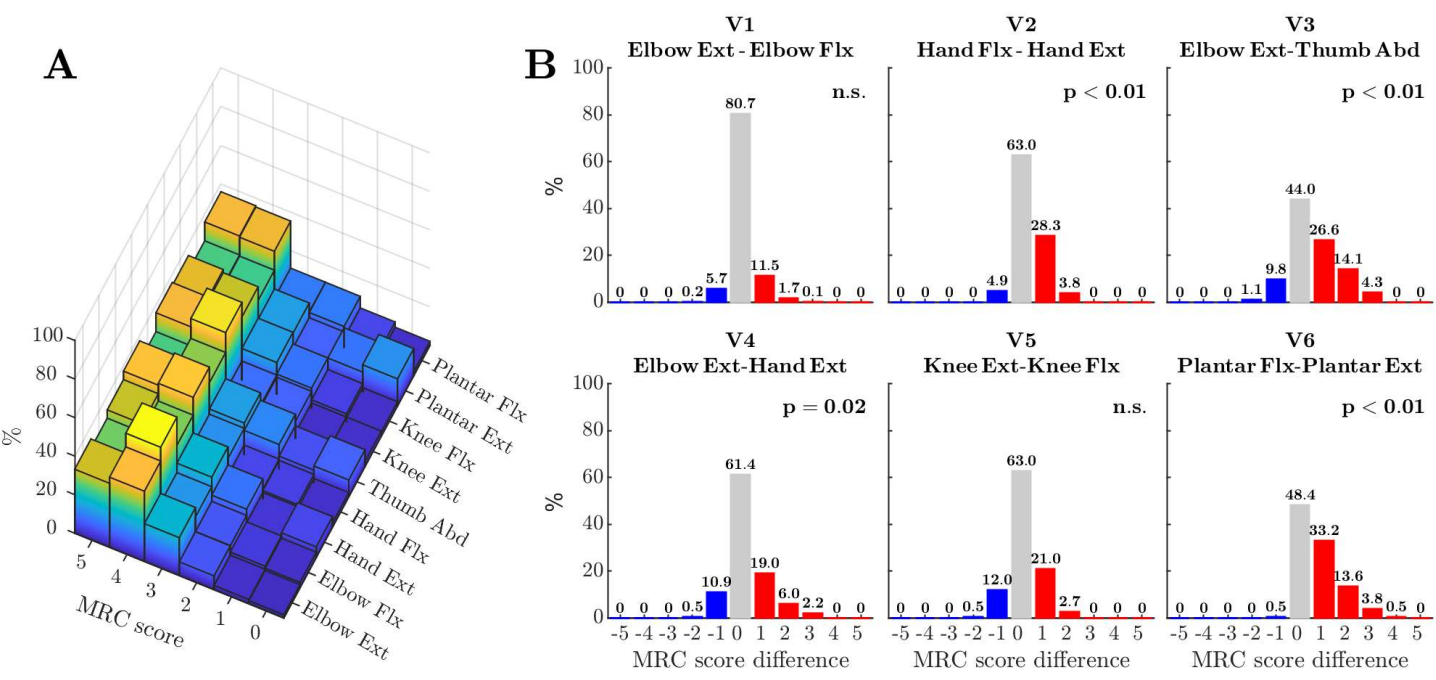

Figure 4. Prospective study in $\mathrm{N}=92$ patients $(\mathrm{N}=184$ limbs). A. Percentages of individual MRC scores for each muscle group. B. Difference in MRC score between pairs of muscles for each muscle group. Bars indicate the percentage distribution of differences in MRC score. See caption accompanying Fig. 2 for further details.

\section{Prospective study - only patients with all extremities affected (subset of 61 patients)}

In this subset of 61 patients, the pattern of paresis was again similar to the main cohort (Fig. 5A) as were the differences between muscle groups (Fig. 5B). The results shown in Fig. 5B demonstrate that the median difference in MRC score was zero for each pair of muscle groups, with the exception of $\mathrm{V} 3$ (elbow extensors - thumb abductors) and 
V6 (plantar flexors - plantar extensors), both median difference: +1 . The differences in groups V2, V3, V4, and V6 were all significant.
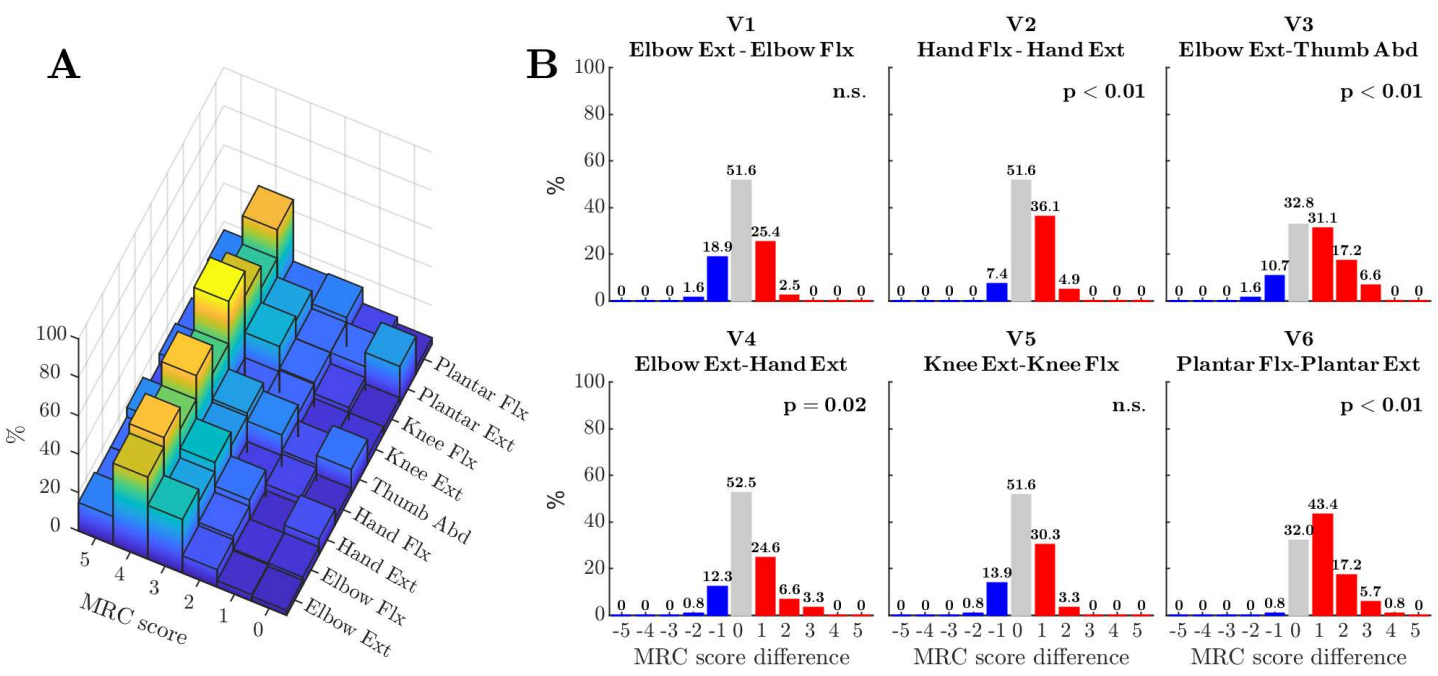

Figure 5. Prospective study: only patients in whom all 4 limbs were affected by disease (subgroup of $\mathrm{N}=61$ patients, $\mathrm{N}=122$ limbs). A. Percentages of individual MRC scores for each muscle group. B. Difference in MRC score between pairs of muscles for each muscle group. Bars indicate the percentage distribution of differences in MRC score. See legend accompanying Fig. 2 for further details.

\section{Second prospective study (54 patients)}

Finally, a second cohort of 54 ALS patients was tested prospectively by an experienced neurologist (ACL). No data were recorded from the knee muscle groups (V5). The pattern of paresis is shown in Fig. 6A and the differences across muscle pairs shown in Fig. 6B. The median difference in MRC score was zero for each pair of muscle groups with the exception of V1 (elbow extensors - elbow flexors) and V3 (elbow extensors - thumb abductors), both median difference: +1 .

Significant differences were found for all pairs of muscle groups tested. We attributed the small differences in the results obtained between the two prospective cohorts to the fact that the patients in this second prospective study were seen as outpatients by the experienced neurologist for a second opinion and, therefore, were in a later stage of the disease. 


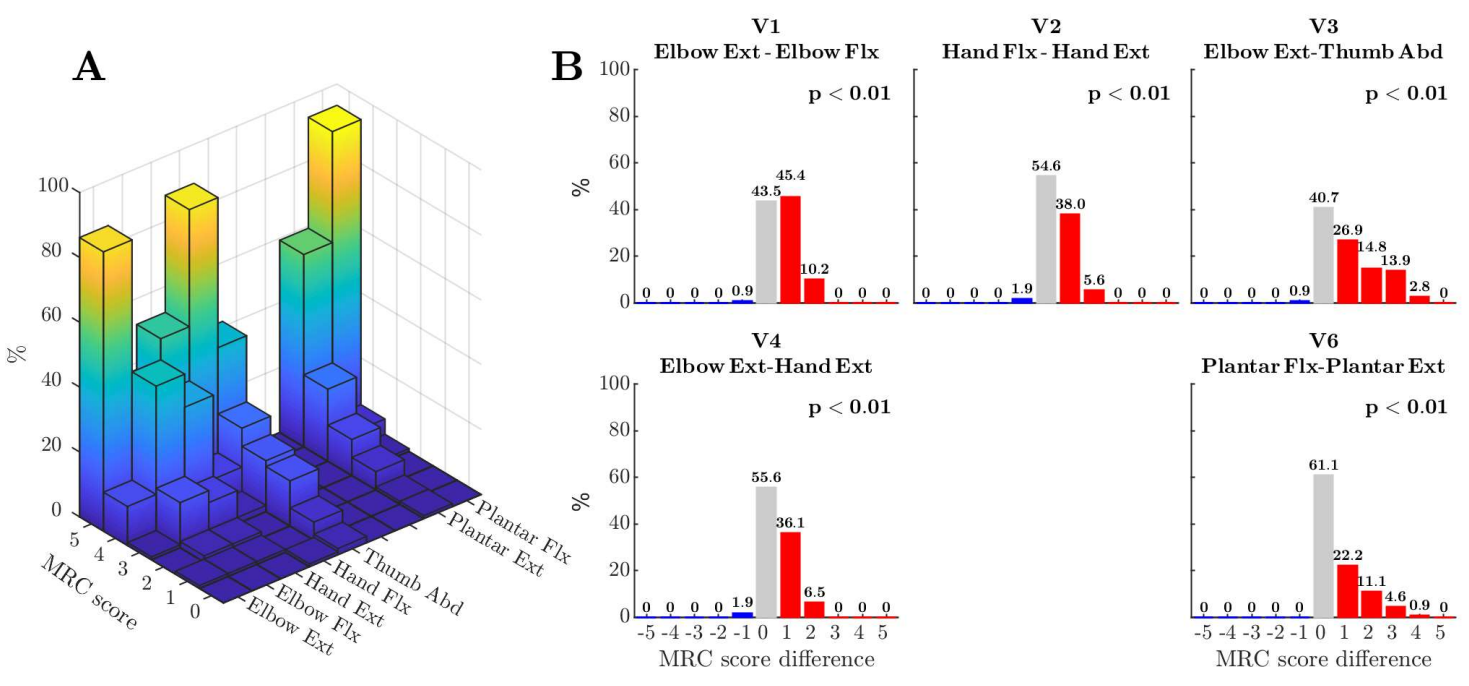

Figure 6. Prospective study in $\mathrm{N}=54$ patients $(\mathrm{N}=108$ limbs). A. Percentages of individual MRC scores for each muscle group. No data was available for the knee flexors or extensors) B. Difference in MRC score between pairs of muscles for each muscle group. Bars indicate the percentage distribution of differences in MRC score. See caption accompanying Fig. 2 for further details.

\section{Discussion}

In this clinical study, we addressed the question whether the pattern of muscle weakness in ALS reflects the known pattern of corticomotoneuronal (CM) influence over different muscle groups in humans and other primates. We were prompted to make this study by other neurophysiological studies implicating the involvement of the $\mathrm{CM}$ projection ${ }^{9,19 \mathrm{~b}}$ and especially by the existence of the split hand syndrome in ALS. ${ }^{26}$, 26a We were also motivated by our own neuropathological findings which support the same conclusion. ${ }^{5-7}$

Analysis of three different patient cohorts showed that the pattern of muscle weakness reflected the pattern of $\mathrm{CM}$ connections. The demographics of our cohorts are valid and not influenced by a selection bias because they largely reflect the demographics in our epidemiological Swabia Registry, which is consistent which the literature and also reflects the demographical changes in highly industrialized Western countries. ${ }^{24}$ 
Our results lend support to neuroanatomical findings that ALS affects long corticofugal pathways and the targets with which they make monosynaptic connections, including $\alpha$-motoneurons. ${ }^{5}$ The findings reported here are also complementary to the previous descriptions of clinical phenomena that have emphasized the particular and early weakness of muscle groups ${ }^{26 \mathrm{~b}, 26 \mathrm{c}}$ over which the CM system has a greater influence ${ }^{9}$, 26,27 as well as the early loss of skilled movements involving these muscle groups. ${ }^{27}$, 27a Our results confirm those of Khalaf et al. (2019), who demonstrated weakness in the biceps compared with triceps brachii (split elbow) in ALS patients. ${ }^{28,28 a}$ In addition, they are consistent with the recent suggestion by Chen et al. that associates the C9ORF72 repeat with the development of lesions in the phylogenetically young $\mathrm{CM}$ connections. ${ }^{29}$ The clinically stereotypical features of ALS, together with the results of studies using non-invasive electrical and magnetic stimulation of the human motor cortex, 19b, 27 may help to establish differential diagnostic criteria, if compared with inclusion body myopathy (where - in contrast to ALS - the flexor muscles of the hand are more affected than the extensors), and with adult spinal muscular atrophy.

How solid is the evidence that the pattern of paresis we observed represents preferential damage to CM pathways? Two major lines of argument exist: First, in early lesion studies performed on non-human primates, Tower suggested that the corticospinal system influences not only hand but also more proximal arm muscles. ${ }^{30}$, 31 Later, Lawrence and Kuypers showed that dexterous finger movements were permanently abolished by bilateral pyramidal tract lesions, and this was attributed to the loss of CM projections. 10, 15, 32, 33 Subsequent electrophysiological studies in nonhuman primates, while again emphasizing the dominant $\mathrm{CM}$ influence over distal hand muscles, have also confirmed effects on more proximal muscles, such as biceps brachii. ${ }^{10,11,34,35}$

Second, transcranial magnetic and electrical stimulation of the motor cortex in humans have added to these results. ${ }^{16-18,36}$ In particular, the work of de Noordhout et al. confirmed the conclusions of non-human primate studies in terms of strong CM actions on intrinsic hand muscles and on the hand/digit extensor muscle, extensor digitorum communis. ${ }^{16}$ There is a graded pattern of $\mathrm{CM}$ influence on upper limb muscles. ${ }^{18}$ Direct electrical stimulation of the human pyramidal tract has been shown to elicit large fast responses in biceps brachii motor units ${ }^{37}$ (see also ${ }^{18,36}$ ). 
Studies of lower limb muscles in humans have demonstrated that plantar extensors receive more direct cortical input than plantar flexors ${ }^{19}, 19 a, 38$ What is still chiefly unknown is the degree of $\mathrm{CM}$ input to the proximal leg muscles. Given the finding here that knee flexors were more affected by the disease process in ALS than knee extensors, it would be worthwhile to test the hypothesis that knee flexors receive more predominantly monosynaptic input from the cortex than the knee extensors. In their electrophysiological studies, Brouwer and Ashby did not see a major difference between the biceps femoris and the vastus medialis with regard to responses from from the motor cortex. ${ }^{19}$ Interestingly, Wartenberg viewed weakness of the knee flexors as belonging to the pattern of 'central' paresis. ${ }^{20} \mathrm{~A}$ recent transcranial magnetic stimulation study showed abnormal motor evoked potentials in both thumb abductors and plantar extensors. ${ }^{39}$

These conclusions are further complemented by recent MRI studies of fractional anisotropy of corticofugal fibers which show that, as a rule, the corticospinal tracts are affected by the disease process, even in progressive muscular atrophy (PMA), a disease traditionally seen by many as a "lower motor neuron" disease. ${ }^{40-42}$ It may be that, in the vast majority of ALS patients, involvement of the corpus callosum linking cortical motor areas and associated clinical phenomena is explained by cortical spreading. ${ }^{43-45}$

During the execution of a skilled movement, the CM system does not act alone but in concert with other descending pathways, many of which receive strong corticofugal projections from the motor areas. ${ }^{11,15,21}$ The clinical pattern of paresis which we have observed may reflect this, in that while many ALS patients showed a clear and statistical bias towards weakness in muscle groups strongly influenced by the CM system, other patients, represented by the large central bins in Figs. 2-6, showed a pattern of weakness that did differ greatly between muscle groups. This might be because these patients were assessed early in the disease process, before the CM system is badly affected, or might result from the involvement of other descending pathways. It might also be due to a more generalized pathology at the level of the $\alpha$ motoneuron. We consider the lack of sufficient knowledge about the pathoanatomy of 
$\alpha$-motoneurons and their relationship to different descending pathways a limiting factor in clarifying this aspect of our results.

Together with neuropathological findings in ALS, the advances discussed above point to the importance of motor cortex and corticospinal tract pathology in different forms of ALS, including primary lateral sclerosis and even the self-limited neurotoxic disease neurolathyrism caused by consumption of Lathyrus sativus, the oldest 'motor neuron disease' on record, described by Hippocrates. ${ }^{5,}$ 7, 8, 46-48

The present study lends further support to the hypothesis raised by neuropathological and neuroanatomical studies that ALS is not a primary neuromuscular disease but a primary cortical disease predominantly affecting long corticofugal axons that secondarily affects the peripheral neuromuscular apparatus. 5,27 If this concept could be translated into differential diagnostic criteria, the clinical diagnosis of sporadic ALS would be greatly facilitated.

\section{Acknowledgements}

The authors wish to thank Mr. David Ewert for technical assistance with the graphics.

\section{Competing interests}

The authors have no current or potential conflicts of interest.

\section{Funding}

This work was funded, in part, by the Virtual Helmholtz Institute for RNA Dysmetabolism in ALS and FTD and the German Research Council (DFG) (LU 336/151)

\section{References}

1 Braak H, Braak E. Neuropathological staging of Alzheimer-related changes. Acta Neuropathol 1991; 82: 239-59.

2 Braak H, Del Tredici K, Rüb U, et al. Staging of brain pathology related to sporadic Parkinson's disease. Neurobiol. Aging 2003; 24: 197-211. 
3 Braak H, Del Tredici K. Neuropathological staging of brain pathology in sporadic Parkinson's disease: Separating the wheat from the chaff. J Parkinsons Dis 2017; 7: S73-S87.

4 Neumann M, Sampathu DM, Kwong LK, et al. Ubiquitinated TDP-43 in frontotemporal lobar degeneration and amyotrophic lateral sclerosis. Science 2006; 314: 130-3.

5 Braak $\mathrm{H}$, Brettschneider J, Ludolph AC, et al. Amyotrophic lateral sclerosis - a model of corticofugal axonal spread. Nat Rev Neurol 2013; 9: 708-14.

6 Braak $\mathrm{H}$, Ludolph AC, Neumann M, et al. Pathological TDP-43 changes in Betz cells differ from those in bulbar and spinal $\alpha$-motoneurons in sporadic amyotrophic lateral sclerosis. Acta Neuropathol 2017; 133: 79-90.

7 Brettschneider J, Del Tredici K, Toledo JB, et al. Stages of pTDP-43 pathology in amyotrophic lateral sclerosis. Ann Neurol 2013; 74: 20-38.

8 Fatima M, Tan R, Halliday GM, et al. Spread of pathology in amyotrophic lateral sclerosis: assessment of phosphorylated TDP-43 along axonal pathways. Acta Neuropathol Commun 2015; 3: 47.

9 Eisen A, Weber M. The motor cortex and amyotrophic lateral sclerosis. Muscle Nerve 2001; 24: 564-79.

10 Porter R, Lemon R. Corticospinal Function and Voluntary Movement. Clarendon Press: Oxford University Press; 1993.

11 Lemon RN. Descending pathways in motor control. Ann Rev Neurosci 2008; 31: 195-218.

12 Lemon RN. The G.L. Brown Prize Lecture. Cortical control of the primate hand. Exp Physiol 1993; 78: 263-301.

13 Maier MA, Illert M, Kirkwood PA, et al. Does a C3-C4 propriospinal system transmit corticospinal excitation in the primate? An investigation in the macaque monkey. $J$ Physiol 1998; 511: 191-212.

14 Phillips CG, Porter R. Corticospinal neurones: Their role in movement. London, New York, and San Francisco: Academic Press; 1993. 
15 Kuypers HGJM. Anatomy of the descending pathways. In: Brookhart JM, Mountcastle VB, Brooks VB, editors. Handbook of Physiology, Sect. 1, Vol. 2. Pt. 1. Bethesda MD: American Physiological Society; 1981, p. 597-666.

16 de Noordhout AM, Rapisarda G, Bogacz D, et al. Corticomotoneuronal synaptic connections in normal man: an electrophysiological study. Brain 1999; 122: 132740.

17 Baldissera F, Cavallari P. Short-latency subliminal effects of transcranial magnetic stimulation on forearm motoneurones. Exp Brain Res 1993; 96: 513-18.

18 Palmer E, Ashby P. Corticospinal projections to upper limb motoneurones in humans. J Physiol 1992; 448: 397-412.

19 Brouwer B, Ashby J. Coricospinal projections to lower limb motoneurons in man. Exp Brain Res 1992; 89: 649-54.

19a. Bawa P, Chalmers GR, Stewart H, Eisen AA. Responses of ankle extensor and flexor motoneurons to transcranial magnetic stimulation. J Neurophysiol 2002; 88: 124-132.

19b. Dharmadasa T, Matamala JM, Howells J, et al. Early focality and spread of cortical dysfunction in amyotrophic lateral sclerosis: A regional study across the motor cortices. Clin Neurophysiol 2020; 131: 958-966.

20 Wartenberg R. Diagnostic tests in neurology; a selection for office use. Chicago IL: Year Books Publishers; 1953. (German: Wartenberg R. Neurologische Untersuchungsmethoden in der Sprechstunde. Stuttgart: Thieme; 1954).

21 Baker SN. The primate reticulospinal tract, hand function and functional recovery. J Physiol 2011; 589(Pt 23): 5603-12.

22 Krakauer JW, Carmichael ST. Broken Movement. The Neurobiology of Motor Recovery After Stroke. Cambridge, MA: MIT Press; 2017.

23 James MA. Use of the Medical Research Council muscle strength grading system in the upper extremity. $J$ Hand Surg Am 2007; 32: 154-6.

24 Rosenbohm A, Peter RS, Erhardt S, et al. Epidemiology of amyotrophic lateral sclerosis in Southern Germany. J Neurol 2017; 264: 749-57. 
25 Brooks BR, Miller RG, Swash M, et al. World Federation of Neurology Research Group on Motor Neuron Diseases. El Escorial revisited: revised criteria for the diagnosis of amyotrophic lateral sclerosis. Amyotroph Lateral Scler Other Motor Neuron Disord 2000; 1: 293-9.

26 Weber M, Eisen A, Stewart H, et al. The split hand in ALS has a cortical basis. $J$ Neurol Sci 2000; 180: 66-70.

26a. Henderson RD, Eisen A. ALS Split phenotypes - To what extent do they exist? Clin Neurophysiol 2020; 131: 847-849.

26b. Oppenheim RW. Lehrbuch der Nervenkrankheiten. Berlin: S. Karger, 1913

26c. Swash M, Burke D, Turner MJ, et al. Occasional essay: Upper motor neuron syndrome in amyotrophic lateral sclerosis. J Neurol Neurosurg Psychiatry 2020; 91: 227-234.

27 Eisen A, Braak $\mathrm{H}$, Del Tredici $\mathrm{K}$, et al. Cortical influences that drive sporadic amyotrophic lateral sclerosis. J Neurol Neurosurg Psychiatry 2017; 88: 917-24.

27a. Eisen A, Turner MR, Lemon RN. Tools and talk: An evolutionary perspective on the functional deficits associated with amyotrophic lateral sclerosis. Muscle Nerve 2014; 49: 469-477.

28 Khalaf R, Martin S, Ellis C, et al. Relative preservation of triceps over biceps strength in upper limb-onset ALS: the "split elbow". J Neurol Neurosurg Psychiatry 2019; 90: 730-733.

28a. Vucic S. Split elbow sign: more evidence for the importance of cortical dysfunction in ALS. J Neurol Neurosurg Psychiatry 2019; 90: 729.

29 Chen Z, Lin K, Macklis J, et al. Proposed association between the hexanucleotide repeat of C9orf72 and opposability index of the thumb. Amyotroph Lateral Scler Frontotemporal Degener 2017; 18: 175-181.

30 Tower SS. Pyramidal lesions in the monkey. Brain 1940; 63: 36-90.

31 Tower SS. The pyramidal tract. In: The Precentral Motor Cortex. Bucy PC, editor. Urbana IL: University of Illinois Press; 1999, p.149-72. 
32 Lawrence DG, Kuypers HG. The functional organization of the motor system in the monkey. I. The effects of bilateral pyramidal lesions. Brain 1968; 91: 1-14.

33 Armand J. The origin, course and terminations of corticospinal fibers in mammals. Prog Brain Res 1982; 57: 329-60.

34 McKiernan BJ, Marcario K, Karrer JH, Cheney PD. Corticomotoneuronal postspike effects in shoulder, elbow, wrist, digit, and intrinsic hand muscles during a reach and prehension task. J Neurophysiol 1998; 80:1961-1980.

35 Clough JFM, Kernell D, Phillips CG. The distribution of monosynaptic excitation from the pyramidal tract and from primary spindle afferents to motoneurones of the baboon's hand and forearm. J Physiol 1988; 198: 145-66.

36 Colebatch JG, Rothwell JC, Day BL, et al. Cortical outflow to proximal arm muscles in man. Brain 1990; 113: 1843-56.

37 Petersen NT, Taylor JL Gandevia SC. The effect of electrical stimulation of the corticospinal tract on motor units of the human biceps brachii. J Physiol 2002; 544(Pt 1): 277-84.

38 Barthélemy D, Grey MJ, Nielsen JB, Bouyer L. Involvement of the corticospinal tract in the control of human gait. Prog Brain Res 2011; 192: 181-97.

39. Menon P, Yianikkas C, Kiernan M, Vucic S. Regional motor cortex dysfunction in amyotrophic lateral sclerosis. Ann Clin Transl Neurol 2019; 6: 1373-82.

40 Kassubek J, Müller H-P, Del Tredici K, et al. Diffusion tensor imaging analysis of sequential spreading of disease in amyotrophic lateral sclerosis confirms patterns of TDP-43 pathology. Brain 2014; 137: 1733-40.

41 Ince PG, Evans J, Knopp M, et al. Corticospinal tract degeneration in the progressive muscular atrophy variant of ALS. Neurology 2003; 60: 1252-8.

42 Rosenbohm A, Müller HP, Hübers A, et al. Corticoefferent pathways in pure lower motor neuron disease: a diffusion tensor imaging study. J Neurol 2016; 263: 24307.

43 Agosta F, Galantucci S, Riva N, et al. Intrahemispheric and interhemispheric structural network abnormalities in PLS and ALS. Hum Brain Mapp 2014; 35: $1710-$ 22. 
44 Kassubek J, Ludolph AC, Müller H-P. Neuroimaging of motor neuron diseases. Ther Adv Neurol Disord 2012; 5: 119-27.

45 Wittstock M, Meister S, Walter U, et al. Mirror movements in amyotrophic lateral sclerosis. Amyotroph lateral scler 2011; 12: 393-7.

46 Wais $\mathrm{V}$, Rosenbohm A, Petri S, et al. The concept and diagnostic criteria of primary lateral sclerosis. Acta Neurol Scand 2016; 136: 204-11.

47 Ludolph AC, Hugon J, Dwivedi MP, et al. Studies on the aetiology and pathogenesis of motor neuron diseases. 1. Lathyrism: clinical findings in established cases. Brain 1987; 110: 149-65.

48 Hugon J, Ludolph A, Roy DN, et al. Studies on the etiology and pathogenesis of motor neuron disease. II. Clinical and electrophysiologic features of pyramidal dysfunction in macaques fed Lathyrus sativus and IDPN. Neurology 1988; 38: 43542.

\section{Figure Legends}

Figure 1. Muscle groups tested (V1-V6). The strength of muscle groups known to be more strongly influenced by $\mathrm{CM}$ projections was compared with those with less direct connections from the motor cortex. Strength of muscles was compared using the MRC score.

Figure 2. Retrospective study in $\mathbf{N}=\mathbf{4 3 6}$ patients ( $\mathrm{N}=\mathbf{8 7 2}$ limbs). A. Percentages of individual MRC scores for each muscle group. Bars indicate the percentage of MRC scores between 0 and 5 . Colour scale is autoscaled to the maximum MRC score across all muscle groups. B. Difference in MRC score between pairs of muscles for each muscle group. Bars indicate the percentage distribution of differences in MRC score. A positive difference (red bars) indicates that the muscle group receiving the less pronounced CM influence (first muscle group listed for each pair) was the stronger. Blue bars indicate in a difference in the opposite direction. The $p$ value derived from the sign test of these differences is given at the top of each panel. 
Figure 3. Retrospective study, only patients in whom all 4 limbs were affected (subset of $\mathrm{N}=\mathbf{2 3 3}$ patients; $\mathrm{N}=\mathbf{1 8 4} \mathrm{limbs}$ ). A. Percentages of individual MRC scores for each muscle group. B. Difference in MRC score between pairs of muscles for each muscle group. Bars indicate the percentage distribution of differences in MRC score. See legend accompanying Fig. 2 for further details.

Figure 4. Prospective study in $\mathrm{N}=\mathbf{9 2}$ patients ( $\mathrm{N}=\mathbf{1 8 4}$ limbs). A. Percentages of individual MRC scores for each muscle group. B. Difference in MRC score between pairs of muscles for each muscle group. Bars indicate the percentage distribution of differences in MRC score. See caption accompanying Fig. 2 for further details.

Figure 5. Prospective study: only patients in whom all 4 limbs were affected by disease (subgroup of $\mathbf{N}=\mathbf{6 1}$ patients, $\mathrm{N}=122$ limbs). A. Percentages of individual MRC scores for each muscle group. B. Difference in MRC score between pairs of muscles for each muscle group. Bars indicate the percentage distribution of differences in MRC score. See legend accompanying Fig. 2 for further details.

Figure 6. Prospective study in $\mathbf{N}=\mathbf{5 4}$ patients ( $\mathrm{N}=108$ limbs). A. Percentages of individual MRC scores for each muscle group. No data was available for the knee flexors or extensors) B. Difference in MRC score between pairs of muscles for each muscle group. Bars indicate the percentage distribution of differences in MRC score. See caption accompanying Fig. 2 for further details. 